I. Kubo and Y. Yokoi

Nagoya Math. J.

Vol. 115 (1989), 139-149

\title{
A REMARK ON THE SPACE OF TESTING RANDOM VARIABLES IN THE WHITE NOISE CALCULUS
}

\author{
IZUMI KUBO AND YOSHITAKA YOKOI \\ Dedicated to Professor Takeyuki Hida on the occasion \\ of his sixtieth birthday
}

\section{$\S 1$. Introduction}

The first author and S. Takenaka introduced the structure of a Gel'fand triplet $\mathscr{H} \subset\left(L^{2}\right) \subset \mathscr{H} *$ into Hida's calculus on generalized Brownian functionals [4-7]. They showed that the space $\mathscr{H}$ of testing random variables has nice properties. For example, $\mathscr{H}$ is closed under multiplication of two elements in $\mathscr{H}$, each element of $\mathscr{H}$ is a continuous functional on the basic space $\mathscr{E}^{*}$, in addition it can be considered as an analytic functional, and moreover $\exp \left[t t_{V}\right]$ ( $\Delta_{V}$ is Volterra's Laplacian) is real analytic in $t \in \boldsymbol{R}$ as a one-parameter group of operators on $\mathscr{H}$, etc.

In this paper, we will prove, by a method different from [4-7], that each element of $\mathscr{H}$ is continuous on the basic space $\mathscr{E}^{*}$ and by using this result we will show that the evaluation map $\delta_{x}: \varphi \mapsto \varphi(x)(x \in \mathscr{E} *)$ belongs to $\mathscr{H}^{*}$. The norm of $\delta_{x}$ will also be estimated.

The fact that $\delta_{x}$ belongs to $\mathscr{H}^{*}$ is very useful in the argument of positive functionals [8].

\section{§ 2. Gel'fand triplets}

Here we will summarize fundamental facts about three Gel'fand triplets $\mathscr{F} \leftrightharpoons \mathscr{F}(0) \subseteq \mathscr{F} *, \exp [\hat{\otimes} \mathscr{E}] \subseteq \exp \left[\hat{\otimes} E_{0}\right] \subseteq \exp \left[\hat{\otimes} \mathscr{E}^{*}\right]$ and $\mathscr{H} \subseteq\left(L^{2}\right)$ $\subset \mathscr{H}^{*}$, which were introduced and discussed in [4-7, 9], for later use. Let $T$ be a separable topological space with a topological Borel field $\mathscr{B}$ and $\nu$ be a $\sigma$-finite measure on $T$ without atoms. We suppose that there exists a Gel'fand triplet (or a rigged Hilbert space) $\mathscr{E} \subset L^{2}(T, \nu) \subset \mathscr{E}^{*}$ (cf. [3]). Namely, the space $\mathscr{E}$ of testing functions on $T$ is topologized by the pro-

Received August 28, 1987. 
jective limit of Hilbert spaces $\left\{E_{p}\right\}_{p \in \boldsymbol{Z}}$ with inner products $\left\{(\xi, \eta)_{p} ; \xi, \eta \in \mathscr{E}\right\}_{p \in \boldsymbol{Z}}$ such that

(G.1) $(\xi, \eta)_{0} \equiv \int_{T} \xi(t) \eta(t) d \nu(t)$,

(G.2) the norms $\left\{\|\xi\|_{p}=\left((\xi, \xi)_{p}\right)^{1 / 2}\right\}_{p \in Z}$ are consistent and increasing,

(G.3) $E_{-p}$ is the dual space of $E_{p}(p \geq 0)$, and

(G.4) for any $p$ there exists $q(>p)$ such that the injection mapping $\iota_{p, q}: E_{q} \rightarrow E_{p}$ is of Hilbert-Schmidt type.

The dual space $\mathscr{E}^{*}$ of $\mathscr{E}$ is the inductive limit of $E_{-p}$ as $p \rightarrow \infty$. We denote by $\langle\cdot, \cdot\rangle$ the canonical bilinear forms between any dual pairs. Then obviously, $\langle\xi, \eta\rangle=(\xi, \eta)_{0}$ holds if $\xi, \eta \in \mathscr{E}$.

Further let us assume the following [A.1] and [A.2].

[A.1] There exists a constant $\rho \in(0,1)$ such that

$$
\rho\|\xi\|_{p+1} \geq\|\xi\|_{p} \quad \text { for any } \xi \in \mathscr{E} \text { and any } p \in Z \text {. }
$$

[A.2] The evaluation map $\delta_{t}: \xi \mapsto \xi(t)$ gives a continuous map $t \mapsto \delta_{t}$ from $T$ into $E_{-1}$ with

$$
\|\delta\|^{2} \equiv \int_{T}\left\|\delta_{t}\right\|_{-1}^{2} d \nu(t)<\infty
$$

Then [A.1] assures suitable analytical properties of nonlinear functionals which appear in these Gel'fand triplets. [A.2] assures that each testing function $\xi(t) \in \mathscr{E}$ is continuous and that the injection $\iota_{0,1}$ is of Hilbert-Schmidt type.

Since $\mathscr{E} \subseteq E_{0}=L^{2}(T, \nu) \subseteq \mathscr{E}^{*}$ is a Gel'fand triplet, by Bochner-Minlos' theorem, we can find a probability measure $\mu$ on $\mathscr{E}^{*}$ such that

$$
\int_{\xi^{*}} \exp [i\langle x, \xi\rangle] d \mu(x)=\exp \left[-\frac{1}{2}\|\xi\|_{0}^{2}\right] \text {. }
$$

Notice that the measure $\mu$ is full on $E_{-1}$, i.e. $\mu\left(E_{-1}\right)=1$ by (2.3). Let us denote $L^{2}\left(\mathscr{E}^{*}, \mu\right)$ simply by $\left(L^{2}\right)$.

Let $E_{p}^{\hat{\otimes} n}$ be the $n$-fold symmetric tensor product of $E_{p}$. By virtue of (G.2), we have natural inclusions $E_{p+1}^{\hat{\otimes} n} \subseteq E_{p}^{\hat{\otimes} n}$. Let $\mathscr{E}^{\hat{\otimes} n}$ denote the projective limit of $E_{p}^{\hat{\otimes} n}$ and $\mathscr{E} * \hat{\otimes} n$ the inductive limit of $E_{-p}^{\hat{\otimes} n}$ as $p \rightarrow \infty$. We always associate the inductive limit convex topology with the inductive limit space. Here we remark the following Lemma, which implies the continuity of the mapping $\mathscr{E}^{*} \ni x \mapsto x^{\hat{\otimes} n} \in \mathscr{E}^{*} \otimes \hat{\otimes}^{n}$. 
Lemma 2.1. Fix a $y \in \mathscr{E}^{*}$, e.g. $y \in E_{-q}$ for some $q \geq 0$, and a neighbourhood $W$ which is given by the absolutely convex envelope of the sets $\left\{z \in E_{-p} ;\|z\|_{-p}<\gamma_{p}\right\}, p \geq q$ with given $\gamma_{p}, 0<\gamma_{p} \leq 1$. Then for any $x \in$ $W+y$, there exists a finite number of positive numbers $\alpha_{p}, q \leq p \leq N$, with $\sum_{p=q}^{N} \alpha_{p} \leq 1$ such that $x^{\hat{\otimes} n}$ is expressed in the form

$$
x^{\hat{\otimes} n}=y^{\hat{\otimes} n}+\sum_{p=q}^{N} v_{n, p} \quad \text { with }\left\|v_{n, p}\right\|_{E-p} \underset{\otimes}{\otimes}<\left(1+\|y\|_{-p}\right)^{n-1} \alpha_{p} \gamma_{p}
$$

for any $n \geq 1$.

Proof. Since any $x \in W+y$ can be written as $x=y+\sum_{p=q}^{N} \alpha_{p} z_{p}$ with $\sum_{p=q}^{N} \alpha_{p} \leq 1, \alpha_{p}>0$ and $\left\|z_{p}\right\|_{-p}<\gamma_{p}$,

$$
v_{n, p} \equiv \sum_{k=1}^{N}\left(\begin{array}{l}
n \\
k
\end{array}\right) \sum_{\max \left(p_{1}, \cdots, p_{k}\right)=p} \alpha_{p_{1}} \cdots \alpha_{p_{k}} z_{p_{1}} \hat{\otimes} \cdots \hat{\otimes} z_{r k} \hat{\otimes} y^{\hat{\otimes}(n-k)}
$$

$p \geq q$, satisfy the requirement.

The orthogonal direct sum

$$
\exp \left[\hat{\otimes} E_{p}\right] \equiv \sum_{n=0}^{\infty} \oplus(n !)^{1 / 2} E_{p}^{\hat{\otimes} n}
$$

with inner product

$$
\left(\left(f_{n}\right)_{n \geq 0},\left(g_{n}\right)_{n \geq 0}\right)_{\exp \left[\hat{\otimes} E_{p}\right]}=\sum_{n=0}^{\infty} n !\left(f_{n}, g_{n}\right)_{E_{p}^{\hat{\otimes}} n}
$$

is called a Fock's space. Its dual space is $\exp \left[\hat{\otimes} E_{-p}\right]$ with the canonical bilinear form

$$
\left\langle\left(G_{n}\right)_{n \geq 0},\left(f_{n}\right)_{n \geq 0}\right\rangle=\sum_{n=0}^{\infty} n !\left\langle G_{n}, f_{n}\right\rangle
$$

for $\left(G_{n}\right)_{n \geq 0} \in \exp \left[\hat{\otimes} E_{-p}\right]$ and $\left(f_{n}\right)_{n \geq 0} \in \exp \left[\hat{\otimes} E_{p}\right], \quad(p \geq 0)$. Again by virtue of (G.2), we have natural inclusions $\exp \left[\hat{\otimes} E_{p+1}\right] \subseteq \exp \left[\hat{\otimes} E_{p}\right]$ for $p \in Z$. We denote by $\exp [\hat{\otimes} \mathscr{E}]$ the projective limit of $\exp \left[\hat{\otimes} E_{p}\right]$ and by $\exp \left[\hat{\otimes} \mathscr{E}^{*}\right]$ the inductive limit of $\exp \left[\hat{\otimes} E_{-p}\right]$ as $p \rightarrow \infty$, respectively.

Proposition 2.2. (a) The triplet $\exp [\hat{\otimes} \mathscr{E}] \subseteq \exp \left[\hat{\otimes} E_{0}\right] \subseteq \exp \left[\hat{\otimes} \mathscr{E}^{*}\right]$ is a Gel'fand triplet.

(b) The mapping from $\mathscr{E}^{*}$ to $\exp [\hat{\otimes} \mathscr{E}]$ defined by

$$
\mathscr{E}^{*} \ni x \longmapsto \exp [\hat{\otimes} x] \equiv \sum_{n=0}^{\infty} \oplus \frac{1}{n !} x^{\hat{\otimes} n} \in \exp \left[\hat{\otimes} \mathscr{E}^{*}\right]
$$

is continuous. 
(c) For $\left(g_{n}\right)_{n \geq 0} \in \exp [\hat{\otimes} \mathscr{E}]$, define a functional $\Psi(x)$ on $\mathscr{E}^{*}$ by

$$
\Psi(x) \equiv \sum_{n=0}^{\infty}\left\langle g_{n}, x^{\hat{\otimes} n}\right\rangle .
$$

Then $\Psi(x)$ is a continuous functicnal on $\mathscr{E}$.

(d) For $\left(G_{n}\right)_{n \geq 0} \in \exp \left[\mathscr{E}^{*}\right]$, define a functional $U(\xi)$ on $\mathscr{E}$ by

$$
U(\xi) \equiv \sum_{n=0}^{\infty}\left\langle G_{n}, \xi^{\hat{\otimes}^{n}}\right\rangle .
$$

Then $U(\xi)$ is a continuous functional on $\mathscr{E}$.

Proof. (a) is seen in [4] by (2.1). (b) Fix a $y \in \mathscr{E}$ and let $q$ be a natural number such that $y \in E_{-q}$. For a given absolutely convex neighbourhood $V$ of the origin of $\exp [\mathscr{E} *]$ of the form

$$
V=\operatorname{conv}\left(\bigcup_{p \geq q}\left\{z ;\|z\|_{\exp [\hat{\otimes} E-p]}<\varepsilon_{p}\right\}\right),
$$

put $\gamma_{p} \equiv \min \left\{\varepsilon_{p} \exp \left[-\left(1+\|y\|_{-p}\right)^{2}\right], 1\right\}$ and let $W$ be the neighbourhood in Lemma 2.1. Then by (2.4), for $x \in W+y$ we have the expression

$$
\exp [\hat{\otimes} x]-\exp [\hat{\otimes} y]=\sum_{q \leq p \leq N}\left(\sum_{n=1}^{\infty} \oplus \frac{1}{n !} v_{n, p}\right)
$$

with norms

$$
\left\|\sum_{n=1}^{\infty} \oplus \frac{1}{n !} v_{n, p}\right\|_{\exp \left[\hat{\otimes} E_{-p}\right]}=\left(\sum_{n=1}^{\infty} \frac{n !}{(n !)^{2}}\left\|v_{n, p}\right\|_{E_{-p}^{2} \hat{\otimes}}^{2}\right)^{1 / 2}<\alpha_{p} \varepsilon_{p} .
$$

Hence $\exp [\hat{\otimes} x] \in V+\exp [\hat{\otimes} y]$ for any $x \in W+y$. Thus (b) is proved. By (b), (c) is obvious since $\left(g_{n}\right)_{n \geq 0}$ is a continuous linear functional on $\exp \left[\hat{\otimes} \mathscr{E}^{*}\right]$ and since $\Psi(x)=\left\langle\left(g_{n}\right)_{n \geq 0}, \exp [\hat{\otimes} x]\right\rangle,(\mathrm{d})$ is easier to prove.

Let $\mathscr{F}\left(\right.$ resp, $\left.\mathscr{F}^{(p)}, \mathscr{F}^{*}\right)$ be the image space of $\exp [\hat{\otimes} \mathscr{E}]$ (resp. $\left.\exp \left[\hat{\otimes} E_{p}\right], \exp [\hat{\otimes} \mathscr{E} *]\right)$ under the mapping (2.8) and introduce a topology from the original space. Then $\mathscr{F}^{(p)}$ is the reproducig kernel Hilbert space with the reproducing kernel $\exp \left[(\xi, \eta)_{-p}\right]$. The following Propositions are shown in [4].

Proposition 2.3. (a) $\mathscr{F}_{F} \subset \mathscr{F}^{(0)} \subset \mathscr{F}^{*}$ is a Gel'fand triplet.

(b) Let $\xi$, and $\zeta$ be in $\mathscr{E}$ and $n, m$ be non-negative integers. Then $\langle\xi, \eta\rangle^{m}$ and $\langle\xi, \zeta\rangle^{n}$ belong to $\mathscr{F}^{(p)}$ and satisfy the equality

$$
\left(\langle\xi, \eta\rangle^{m},\langle\xi, \zeta\rangle^{n}\right)_{\mathcal{F}(p)}=\delta_{m, n} n !(\eta, \zeta)_{p}^{n} \quad \text { for any } p \in Z .
$$


Proposition 2.4. For each fixed $\xi \in \mathscr{E}$, write

$$
f(\xi)=f(\xi ; x) \equiv \exp \left[\langle x, \xi\rangle-\frac{1}{2}\|\xi\|_{0}^{2}\right] .
$$

Then the mapping $\mathscr{S}$ defined by

$$
(\mathscr{S} \varphi)(\xi) \equiv \int_{\mathscr{\theta}^{*}} \varphi^{\prime}(x) f(\xi, x) d \mu(x)=\int_{\sigma^{*}} \varphi(x+\xi) d \mu(x)
$$

is an isomorphism from $\left(L^{2}\right)$ onto $\mathscr{F}^{(0)}$. Especially,

$$
(\mathscr{S} f(\eta))(\xi)=\exp [\langle\eta, \xi\rangle] \quad \text { for any } \xi, \eta \in \mathscr{E}
$$

and

$$
\mathscr{S}: H_{n}\left(\langle x, \eta\rangle ;\|\eta\|^{2}\right) \longmapsto\langle\xi, \eta\rangle^{n},
$$

where $H_{n}(z ; \gamma)(n=0,1,2, \cdots)$ are the Hermite polynomials with parameter $\gamma$ defined by the generating function $\exp \left[\omega z-\frac{\gamma}{2} \omega^{2}\right]$;

$$
\sum_{n=0}^{\infty} \frac{1}{n !} \omega^{n} H_{n}(z ; \gamma) \equiv \exp \left[\omega z-\frac{\gamma}{2} \omega^{2}\right] \text {. }
$$

Put $\mathscr{H}^{(p)} \equiv \mathscr{S}^{-1}(\mathscr{F}(p))$ for $p \geq 0$ and $\mathscr{H} \equiv \mathscr{S}^{-1}(\mathscr{F})$ and introduce inner products by

$$
(\varphi, \psi)_{\mathscr{H}(p)} \equiv(\mathscr{S} \varphi, \mathscr{S} \psi)_{\mathscr{F}(p)}
$$

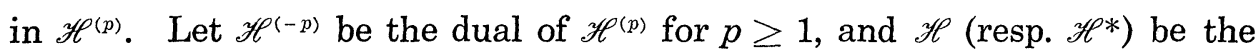
projective (resp. inductive) limit of $\mathscr{H}^{(p)}$. We call $\mathscr{H}$ the space of testing random variables and $\mathscr{H}^{*}$ the space of generalized random variables.

Proposition 2.5. For any $\xi \in \mathscr{E}, f(\xi ; x)$ is in $\mathscr{H}$ and the mapping $\mathscr{S}$ is extended on $\mathscr{H}^{*}$ by

$$
(\mathscr{S} \Psi)(\xi)=\langle\Psi(x), f(\xi ; x)\rangle .
$$

Then $\mathscr{S}$ gives the isomorphism from $\mathscr{H} \subseteq\left(L^{2}\right) \subseteq \mathscr{H}^{*}$ to $\mathscr{F} \subseteq \mathscr{F}^{(0)} \subseteq \mathscr{F}^{*}$. Namely, $\mathscr{H}^{(p)}$ is isomorphic to $\mathscr{F}^{(p)}$ through $\mathscr{S}$ for any $p \in Z$.

Proposition 2.6. For $p \geq 0$, the isomorphism

$$
\exp \left[\hat{\otimes} E_{p}\right] \ni\left(f_{n}\right)_{n \geq 0} \longmapsto \varphi \in \mathscr{H}^{(p)}
$$

is given by

$$
\varphi=\sum_{n=0}^{\infty} I_{n}\left(f_{n}\right), \quad\|\varphi\|_{\mathscr{\mathscr { C }}(p)}=\left\|\left(f_{n}\right)_{n \geq 0}\right\|_{\exp \left[\hat{\otimes} E_{p}\right]}
$$


where $I_{n}\left(f_{n}\right)$ is the multiple Wiener-Itô integral

$$
I_{n}\left(f_{n}\right)=\int \cdots \int_{T^{n}} f_{n}\left(t_{1}, \cdots, t_{n}\right) W\left(d t_{1}\right) \cdots W\left(d t_{n}\right)
$$

with respect to the Gaussian white noise $W(d t)$ given by the relation

$$
\langle x, \xi\rangle=\int_{T} \xi(t) W(d t, x) \text { a.s. } x \in \mathscr{E}^{*}(\mu) .
$$

\section{$\S 3$. The space $\mathscr{H}$ of testing ramdom variables}

In [4-7], it was shown that the multiplication $\varphi, \psi \mapsto \varphi \cdot \psi$ is continuous as the mapping from $\mathscr{H} \times \mathscr{H}$ into $\mathscr{H}$. Further each element of $\varphi \in \mathscr{H}$ is continuous functional on $\mathscr{E}^{*}$. More surprising thing is that each $U(\xi) \in \mathscr{F}$ can be extended to a continuous functional $\tilde{U}(x)$ on $\mathscr{E}^{*}$ and the class $\{\tilde{U}(x) ; U(\xi) \in \mathscr{F}\}$ coincides with $\mathscr{H}$. Those results were proved in a very complicated way with the help of Volterra's Laplacian.

Here we prove the continuity in $x \in \mathscr{E}^{*}$ for every functional $\varphi(x) \in \mathscr{H}$ and the continuity of the evaluation map:

$$
\delta_{x}: \mathscr{H} \ni \varphi \longmapsto \varphi(x) \in \boldsymbol{R},
$$

directly by using basic results.

Firstly, we prove that the multiple Wiener-Itô integral $I_{n}\left(f_{n}\right)$ has a continuous version as a functional on $\mathscr{E}^{*}$ if $f_{n}$ is a good function.

THEOREM 3.1. For $f_{n} \in \mathscr{E}^{\hat{\otimes} n}$,

$$
I_{n}\left(f_{n}\right)(x)=\sum_{k=0}^{[n / 2]}(-1)^{k} \frac{n ! 2^{-k}}{(n-2 k) ! k !}\left\langle x^{\hat{\otimes}(n-2 k)}, f_{n \mid n-2 k}\right\rangle \quad \text { a.s. } x \in \mathscr{E}^{*},
$$

where

$$
\begin{aligned}
& f_{n \mid n-2 k}\left(t_{1}, \cdots t_{n-2 k}\right) \\
& \quad \equiv \int \cdots \int_{T^{k}} f_{n}\left(t_{1}, \cdots, t_{n-2 k}, u_{1}, u_{1}, \cdots, u_{k}, u_{k}\right) d \nu\left(u_{1}\right) \cdots d \nu\left(u_{k}\right) .
\end{aligned}
$$

Proof. We denote by $\mathscr{I}_{n}\left(f_{n}\right)$ the right hand side of (3.2) for $f_{n} \in \mathscr{E}^{\hat{\otimes}}$. Then it is a continuous (non-linear) functional of $x \in \mathscr{E}^{*}$ because of Lemma 2.1 and of the following estimation:

$$
\begin{aligned}
& \left\|f_{n \mid n-2 k}\right\|_{E_{p}^{\hat{\otimes}(n-2 k)}} \\
& \quad \leqq \int \cdots \int_{T^{k}}\left\|f_{n}\left(t_{1}, \cdots, t_{n-2 k}, u_{1}, u_{1}, \cdots, u_{k}, u_{k}\right)\right\|_{E_{p}^{\hat{\otimes}}(n-2 k)} d \nu\left(u_{1}\right) \cdots d \nu\left(u_{k}\right)
\end{aligned}
$$




$$
\begin{aligned}
& \leq \int \cdots \int_{T^{k}}\left\|f_{n}\right\|_{E_{p}^{\hat{\otimes}} n}\left\|\delta_{u_{1}}\right\|_{-1}^{2} \cdots\left\|\delta_{u_{k}}\right\|_{-1}^{2} \rho^{2(p-1) k} d \nu\left(u_{1}\right) \cdots d \nu\left(u_{k}\right) \\
& \leq\left\|f_{n}\right\|_{E_{p}^{\hat{\otimes}} n}\left(\|\delta\| \rho^{p-1}\right)^{2 k}
\end{aligned}
$$

Consequently, for $x \in E_{-p}$, we have

$$
\begin{aligned}
\left|\mathscr{I}_{n}\left(f_{n}\right)(x)\right| & \leq \sum_{k=0}^{[n / 2]} \frac{n ! 2^{-k}}{(n-2 k) ! k !}\|x\|_{-p}^{n-2 k}\left\|f_{n \mid n-2 k}\right\|_{E_{p}^{\hat{\otimes}(n-2 k)}} \\
& \leq \sum_{k=0}^{[n / 2]} \frac{n ! 2^{-k}}{(n-2 k) ! k !}\|x\|_{-p}^{n-2 k}\left(\|\delta\| \rho^{p-1}\right)^{2 k}\left\|f_{n}\right\|_{E_{p}^{\hat{\otimes} n}} \\
& \leq \sqrt{n !} \sum_{k=0}^{n} \frac{n !}{(n-k) ! k !}\|x\|_{-p}^{n-k}\left(\|\delta\| \rho^{p-1}\right)^{k}\left\|f_{n}\right\|_{E_{p}^{\hat{\otimes}} n} \\
& \leq \sqrt{n !}\left(\|x\|_{-p}+\|\delta\| \rho^{p-1}\right)^{n}\left\|f_{n}\right\|_{E_{p}^{\hat{\otimes}} n},
\end{aligned}
$$

by $2^{-k} / k !=(2 k-1) ! ! /(2 k) ! \leq \sqrt{n !} /(2 k) !$ for $2 k \leq n$. Since $\mathscr{I}_{n}\left(f_{n}\right)$ is linear in $f_{n}, \mathscr{I}_{n}\left(f_{n}^{(j)}\right)$ converges to $\mathscr{I}_{n}\left(f_{n}\right)$ uniformly on any bounded set $B$ of $\mathscr{E}^{*}$, if $f_{n}^{(j)} \rightarrow f_{n}$ in $\mathscr{E}^{\hat{\otimes} n}$.

First consider the case $f_{n}=\eta\left(t_{1}\right) \cdots \eta\left(t_{n}\right)$. Then the equality $I_{n}\left(f_{n}\right)=$ $H_{n}\left(\langle x, \eta\rangle,\|\eta\|_{0}^{2}\right)$ is well known (actually it is shown by Propositions 2.4 and 2.6). Since the equality

$$
\begin{aligned}
\left\langle x^{\hat{\otimes}(n-2 k)}, f_{n \mid n-2 k}\right\rangle & =\left\langle x^{\hat{\otimes}(n-2 k)},\|\eta\|_{0}^{2 k} \eta\left(t_{1}\right) \cdots \eta\left(t_{n-2 k}\right)\right\rangle \\
& =\|\eta\|_{0}^{2 k}\langle x, \eta\rangle^{n-2 k}
\end{aligned}
$$

holds, (3.2) is obvious in this case by the formula of the Hermite polynomials;

$$
H_{n}(z ; \gamma)=\sum_{k=0}^{[n / 2]}(-1)^{k} \frac{n !(\gamma / 2)^{k}}{(n-2 k) ! k !} z^{n-2 k} \quad \text { (see p. } 193 \text { [11]). }
$$

For a general $f_{n}$ in $\mathscr{E}^{\otimes} n$, there exists a sequence of the form $\left\{f_{n}^{(j)}=\right.$ $\left.=\sum_{l} c_{j, l}\left(\eta_{l}^{(j)}\right)^{\hat{\otimes} n}\right\}_{j=1}^{\infty}$ which converges to $f_{n}$ in $\mathscr{E}^{\hat{\otimes} n}$. Then $I_{n}\left(f_{n}^{(j)}\right)=\mathscr{I}\left(f_{n}^{(j)}\right)$ holds a.s. $x \in \mathscr{E}^{*}$ and $\mathscr{I}_{n}\left(f_{n}^{(j)}\right)$ converges to $\mathscr{I}_{n}\left(f_{n}\right)$ for every $x \in \mathscr{E}^{*}$. Since

$$
\left\|I_{n}\left(f_{n}^{(j)}\right)-I_{n}\left(f_{n}\right)\right\|_{\left(L^{2}\right)}=\sqrt{n !}\left\|f_{n}^{(j)}-f_{n}\right\|_{E_{0}^{\hat{\otimes}} n},
$$

a suitable subsequence of $I_{n}\left(f_{n}^{(j)}\right)$ converges to $I_{n}\left(f_{n}\right)$ a.s. This implies that $I_{n}\left(f_{n}\right)=\mathscr{I}_{n}\left(f_{n}\right)$ a.s. $x \in \mathscr{E}^{*}$.

Now we are ready to prove our main theorem:

THEOREM 3.2. For any $\varphi \in \mathscr{H}, \varphi$ has a continuous version $\varphi(x)$ and it is bounded on each bounded set of $\mathscr{E}^{*}$. Moreover the evaluation map 
$\delta_{x}: \varphi \rightarrow \varphi(x)$ is a continuous linear functional on $\mathscr{H}$, i.e., $\delta_{x} \in \mathscr{H}^{*}$ for any $x \in \mathscr{E}^{*}$.

Proof. For $\varphi \in \mathscr{H}$, let $\left(f_{n}\right)_{n \geq 0}$ be the element of $\exp [\hat{\otimes} \mathscr{E}]$ satisfying (2.15) in Proposition 2.6. Put

$$
g_{m} \equiv \sum_{k=0}^{\infty}(-1)^{k} \frac{(m+2 k) ! 2^{-k}}{m ! k !} f_{m+2 k \mid m} .
$$

Then $\left(g_{m}\right)_{m \geq 0}$ belongs to $\exp [\hat{\otimes} \mathscr{E}]$, because

$$
\begin{aligned}
\|\left(g_{m}\right)_{m} & \geq 0\left\|_{\exp \left[\hat{\otimes} E_{-p}\right]} \leq \sum_{m=0}^{\infty} \sqrt{m !}\right\| g_{m} \|_{E_{-p}^{\hat{\otimes} m}} \\
& \leq \sum_{m=0}^{\infty} \sqrt{m !}\left(\sum_{k=0}^{\infty} \frac{(m+2 k) ! 2^{-k}}{m ! k !}\left(\|\delta\| \rho^{p-1}\right)^{2 k}\left\|f_{m+2 k}\right\|_{E_{-p}^{\hat{\otimes}}(m+2 k)}\right) \\
& \leq \sum_{n=0}^{\infty} \sum_{k=0}^{[n / 2]} \frac{\sqrt{n !} 2^{-k}}{\sqrt{(n-2 k)} k !}\left(\|\delta\| \rho^{p-1}\right)^{2 k} \sqrt{n !}\left\|f_{n}\right\|_{E_{-p-r}^{\hat{\otimes}} n} \rho^{r n} \\
& \leq \sum_{n=0}^{\infty} \sum_{k=0}^{n} \frac{\sqrt{n !}}{\sqrt{(n-k)} k !}\|\delta\|^{k} \sqrt{n !}\left\|f_{n}\right\|_{E_{-p-r}^{\hat{\otimes}} n} \rho^{r n} \\
& \leq \sum_{n=0}^{\infty}(1+\|\delta\|)^{n} \rho^{r n} \sqrt{n !}\left\|f_{n}\right\|_{E_{-p-r}^{\hat{\otimes}} n} \\
& \leq\left(1-(1+\|\delta\|)^{2} \rho^{2 r}\right)^{-1 / 2}\left\|\left(f_{n}\right)_{n \geq 0}\right\|_{\exp \left[\hat{\otimes}_{E-p-r}\right]}
\end{aligned}
$$

for sufficiently large $r$ as $(1+\|\delta\|) \rho^{r}<1$, by $\sqrt{(2 k) !} \leq 2^{k} k$ ! and $1 \leq$ $\frac{n !}{(n-k) ! k !}$. By Theorem 3.1 and the definition of $\mathscr{I}_{n}\left(f_{n}\right)$, we see that

$$
\tilde{\varphi}(x) \equiv \sum_{n=0}^{\infty} \mathscr{I}_{n}\left(f_{n}\right)(x)=\left\langle\left(g_{m}\right)_{m \geq 0}, \exp [\hat{\otimes} x]\right\rangle
$$

and $\varphi(x)=\tilde{\varphi}(x)$ a.s. $\mu$. By Proposition 2.2 (c), $\tilde{\varphi}(x)$ is a continuous functional on $\mathscr{E}^{*}$. By (3.5),

$$
|\tilde{\varphi}(x)| \leq\left|\sum_{n=0}^{\infty} \mathscr{I}_{n}\left(f_{n}\right)(x)\right| \leq\left(1-\left(\|x\|_{-p}+\|\delta\| \rho^{p-1}\right)^{2}\right)^{-1 / 2}\|\varphi\|_{\mathscr{P}(p)}
$$

holds for sufficiently large $p$ as $\|x\|_{-p}+\|\delta\| \rho^{p-1}<1$. This shows that the evaluation map $\delta_{x}$ belongs to $\mathscr{H}^{*}$.

From now on, $\varphi(x)$ (for $\varphi \in \mathscr{H}$ ) is always considered as the continuous version.

\section{$\S$ 4. The evaluation map $\delta_{x}$}

We have seen that $\delta_{y}$ belongs to $\mathscr{H}^{*}$, if $y \in \mathscr{E}^{*}$. Therefore $\delta_{y}$ must belong to $\mathscr{H}^{(-p)}$ for some $p=p(y) \geq 0$ and its image under $\mathscr{S}$ can be 
observed. By (2.14) in Proposition 2.5, we have

$$
\left(\mathscr{S} \delta_{y}\right)(\xi)=\left\langle\delta_{y}, f(\xi ; \cdot)\right\rangle=f(\xi ; y) \quad \text { for } \xi \in \mathscr{E} .
$$

Since $\mathscr{S}$ is an isomorphism from $\mathscr{H}^{(-p)}$ to $\mathscr{F}^{(-p)}$, we can estimate the norm of $\delta_{y}$ by computing $\|f(\xi ; y)\|_{\mathscr{F}(-p)}$ directly.

Suppose that $y \in E_{-p}, p \geq 1$. Since the injection $\iota_{0, p}$ is of HilbertSchmidt type, there exists a c.o.n.s. $\left\{\zeta_{j}\right\}_{j=1}^{\infty}$ of $E_{0}$ such that $\left\{\zeta_{j}\right\}_{j=1}^{\infty} \subset E_{p}$ and $\sum_{j=1}^{\infty} \lambda_{j}^{2}<\infty$ for $\lambda_{j}^{2} \equiv\left\|\zeta_{j}\right\|_{-p}^{2}$. For $\xi \in \mathscr{E}$, we have

$$
\begin{aligned}
f(\xi ; y) & =\exp \left[\langle y, \xi\rangle-\frac{1}{2}\|\xi\|_{0}^{2}\right]=\sum_{j=1}^{\infty}\left(\sum_{n=0}^{\infty} \frac{1}{n !}\left\langle\zeta_{j}^{\hat{\otimes} n}, \xi^{\hat{\otimes} n}\right\rangle H_{n}\left(\left\langle y, \zeta_{j}\right\rangle\right)\right) \\
& =\sum_{n=0}^{\infty} \sum_{n=n_{1}+\cdots+n_{j}+\cdots} \prod_{j=1}^{\infty} \frac{1}{n_{j} !}\left\langle\zeta_{j}^{\hat{\otimes} n_{j}}, \xi^{\hat{\otimes} n_{j}}\right\rangle H_{n_{j}}\left(\left\langle y, \zeta_{j}\right\rangle\right) .
\end{aligned}
$$

Hence we have, for $y$ any $z \in E_{-p}$,

$$
\begin{aligned}
(f(\cdot ; y), f(\cdot ; z))_{\mathscr{F}(-p)} & \\
= & \sum_{n=0}^{\infty} \sum \prod_{j=1}^{\infty} \frac{1}{n_{j} !} \lambda_{j}^{2 n_{j}} H_{n_{j}}\left(\left\langle y, \zeta_{j}\right\rangle\right) \cdot H_{n_{j}}\left(\left\langle z, \zeta_{j}\right\rangle\right) \\
= & \prod_{j=1}^{\infty}\left(\sum_{n=0}^{\infty} \frac{1}{n !} \lambda_{j}^{2 n} H_{n}\left(\left\langle y, \zeta_{j}\right\rangle\right) \cdot H_{n}\left(\left\langle z, \zeta_{j}\right\rangle\right)\right) \\
= & \prod_{j=1}^{\infty}\left(1-\lambda_{j}^{4}\right)^{-1 / 2} \\
& \times \prod_{j=1}^{\infty} \exp \left[-\frac{1}{2} \frac{\lambda_{j}^{4}\left\langle y, \zeta_{j}\right\rangle^{2}-2 \lambda_{j}^{2}\left\langle y, \zeta_{j}\right\rangle\left\langle z, \zeta_{j}\right\rangle+\lambda_{j}^{4}\left\langle z, \zeta_{j}\right\rangle^{2}}{1-\lambda_{j}^{4}}\right] \\
\leq & \prod_{j=1}^{\infty}\left(1-\lambda_{j}^{4}\right)^{-1 / 2} \exp \left[\frac{1}{2}\left(\|y\|_{-p}^{2}+\|z\|_{-p}^{2}\right)\right]
\end{aligned}
$$

by Proposition 2.3 and the formula

$$
\sum_{n=0}^{\infty} \frac{t^{n}}{n !} H_{n}(u) H_{n}(v)=\left(1-t^{2}\right)^{-1 / 2} \exp \left[-\frac{1}{2} \frac{t^{2} u^{2}-2 t u v+t^{2} v^{2}}{1-t^{2}}\right]
$$

with $H_{n}(u)=H_{n}(u ; 1)$ (see [11] p. 194]. In particular,

$$
\begin{aligned}
\|f(\cdot ; y)\|_{\mathscr{F}^{(p)}}^{2} & =\prod_{j=1}^{\infty}\left(\left(1-\lambda_{j}^{4}\right)^{-1 / 2} \exp \left[\frac{\lambda_{j}^{2}\left\langle y, \zeta_{j}\right\rangle^{2}}{1+\lambda_{j}^{2}}\right]\right) \\
& \leq \prod_{j=1}^{\infty}\left(1-\lambda_{j}^{4}\right)^{-1 / 2} \exp \left[\|y\|_{-p}^{2}\right] .
\end{aligned}
$$

Summarizing the above computations, we have:

THEOREM 4.1. The generalized random variable $\delta_{y}$ has the following 
properties;

(a) $\left(\mathscr{S} \delta_{y}\right)(\xi)=f(\xi ; y)=\exp \left[\langle y, \xi\rangle-\frac{1}{2}\|\xi\|_{0}^{2}\right]$,

(b) $\left(\delta_{y}, \delta_{z}\right)_{\mathscr{H}(-p)}=\prod_{j=1}^{\infty}\left(1-\lambda_{j}^{4}\right)^{-1 / 2}$

$$
\times \prod_{j=1}^{\infty} \exp \left[-\frac{1}{2} \frac{\lambda_{j}^{4}\left\langle y, \zeta_{j}\right\rangle^{2}-2 \lambda_{j}^{2}\left\langle y, \zeta_{j}\right\rangle\left\langle z, \zeta_{j}\right\rangle+\lambda_{j}^{4}\left\langle z, \zeta_{j}\right\rangle^{2}}{1-\lambda_{j}^{4}}\right],
$$

(c) $\left\|\delta_{y}\right\|_{\mathscr{e}(-p)} \leq \exp \left[\frac{1}{2}\left\|\varepsilon_{0, p}\right\|_{H . S .}^{2}\right] \exp \left[\frac{1}{2}\|y\|_{-p}^{2}\right] \quad$ if $y \in E_{-p}$,

(d) $\int_{\epsilon^{*}}\left\|\delta_{y}\right\|_{\left.\mathscr{e}^{(}-p\right)}^{2} d \mu(y)=\left\|\iota_{\left(L^{2}\right), \mathscr{e}(p)}\right\|_{H . S .}^{2}$.

Proof. The only thing we still have to prove is (d). By (2.2) the injection $\iota_{0,1}$ from $E_{1}$ into $E_{0}$ is of Hilbert-Schmidt type. By Sazonov's theorem, the support of the measure $\mu$ is $E_{-1}$. Hence the integral in (d) is taken over $E_{-1}$. Since $\left\{\left\langle y, \zeta_{j}\right\rangle ; j=1,2, \cdots\right\}$ are independent of each other with respect to $\mu$, we can easily calculate;

$$
\int_{E_{-1}}\left\|\delta_{\xi^{\prime}}\right\|_{\mathscr{P}(-p)}^{2} d \mu(y)=\prod_{j=1}^{\infty}\left(1-\lambda_{j}^{2}\right)^{-1} .
$$

The left hand side is equal to the Hilbert-Schmidt operator norm of the injection $\iota_{\left(L^{2}\right), \mathscr{e}(p)}$ by the proof of Proposition 3.6 in [9].

In [7], the renormalization : : has been introduced. By the notation used in it we may write

$$
\delta_{y}(x)=: \exp \left[\langle y, x\rangle \cdot-\frac{1}{2} \int_{T}(x(t) \cdot)^{2} d \nu(t)\right]: 1,
$$

because the right hand side is defined by

$$
\mathscr{S}^{-1}\left(\exp \left[\langle y, \xi\rangle-\frac{1}{2} \int_{T} \xi(t)^{2} d \nu(t)\right]\right) .
$$

Acknowlengement. The authors give their hearty thanks to the referee for his suggestions to improve the manuscript.

\section{REFERENCES}

[1] Hida, T., Analysis of Brownian functionals, Carleton Math. Lec. Notes, No.13, 2nd Ed. (1978).

[ 2 ] Gel'fand, I. M. and Shilov, G. E., Generalized functions, Vol. 2. Academic Press.

[ 3 ] Gel'fand, I. M. and Vilenkin, N. Ya., Generalized functions, Vol. 4. Academic Press. 
[4] Kubo, I. and Takenaka, S., Calculus on Gaussian white noises I, Proc. Japan Acad., 56, Ser. A, No. 8, (1980), 376-380.

[ 5 ] - Calculus on Gaussian white noises II, Proc. Japan Acad., 56, Ser. A, No. 9, (1980), 411-416.

[6] - Calculus on Gaussian white noises III, Proc. Japan Acad., 57, Ser. A, No. 9 (1981) , 433-437.

[ 7 ] - Calculus on Gaussian white noises IV, Proc. Japan Acad., 58, Ser. A, No. 9 (1982), 186-189.

[8] Yokoi, Y., Private notes for positivity of generalized Brownian functionals.

[9] Ito, Y. and Kubo, I., Calculus on Gaussian and Poisson white noises, (submitted to Nagoya Math. J.).

[10] Kuo, H.-H., Gaussian measures in Banach spaces, Lect. Notes in Math. Vol. 463, Springer-Verlag, (1975).

[11] Erdélyi, A., Higher transcendental functions, Vol. 2 (1953), McGraw-Hill.

I. Kubo

Faculty of Integrated Arts and Sciences

Hiroshima University

Hiroshima 730, Japan

Y. Yokoi

Department of Mathematics

Faculty of General Education

Kumamoto University

Kumamoto 860, Japan 